\title{
Experimental study of application of molecules with a cyclic head group containing a free radical as organic friction modifiers
}

\author{
Xiaowei ZHANG*, Masaki TSUKAMOTO**, Hedong ZHANG**, \\ Yasunaga MITSUYA***, Shintaro ITOH**** and Kenji FUKUZAWA**** \\ * School of Electromechanical Engineering, Guangdong University of Technology \\ 100 Waihuan Xi Road, Panyu District, Guangzhou 510006, China \\ ** Department of Complex Systems Science, Graduate School of Informatics, Nagoya University \\ Furo-cho, Chikusa-ku, Nagoya 464-8601, Japan \\ E-mail: zhang@i.nagoya-u.ac.jp \\ *** Nagoya Industrial Science Research Institute \\ Noa Yotsuya Building 2F, Yotsuya-Douri 1-13, Chikusa-ku, Nagoya 464-0819, Japan \\ ${ }^{* * * *}$ Department of Micro-Nano Systems Engineering, Graduate School of Engineering, Nagoya University \\ Furo-cho, Chikusa-ku, Nagoya 464-8603, Japan
}

Received: 9 September 2019; Revised: 24 January 2020; Accepted: 18 March 2020

\begin{abstract}
The optimal molecular design of organic friction modifier (OFM) additives allows for a reduction in the friction and wear in mechanical systems, and thus, an improvement in the energy efficiency and reduction in $\mathrm{CO}_{2}$ emissions. In this study, we synthesized $\mathrm{N}-(2,2,6,6$-tetramethyl-1-oxyl-4-piperidinyl)dodecaneamide, referred to as $\mathrm{C}_{12}$ TEMPO, which has a large and rigid cyclic head group containing a free radical, to explore its application as a new type of OFM. We also selected stearic acid and stearonitrile, which possess small head groups without free radicals but an identical tail structure and approximately the same total length as $\mathrm{C}_{12} \mathrm{TEMPO}$, to clarify the effect of head groups. The three types of OFMs were added separately into polyalphaolefin oil at $1 \mathrm{wt} . \%$, and their lubrication performance was measured with a pin-on-disk type friction tester under varied normal loads or sliding speeds. $\mathrm{C}_{12}$ TEMPO exhibited the highest load-carrying capacity, up to roughly thrice that of stearic acid and stearonitrile. Additionally, $\mathrm{C}_{12}$ TEMPO gave rise to more stable sliding than stearic acid, as confirmed from comparison of instantaneous friction coefficients. These results suggested that $\mathrm{C}_{12}$ TEMPO could form an effective boundary film that was robust to heavy-loaded sliding. The reason for this could be attributed to not only the strong surface interaction owing to the free radical but also to the rigidity of the cyclic structure of the head group in $\mathrm{C}_{12} \mathrm{TEMPO}$. The results obtained when varying the sliding speed suggested that the efficiency to form the boundary film followed the order: stearic acid $>\mathrm{C}_{12} \mathrm{TEMPO}>$ stearonitrile. We inferred that the relatively large size of the head group of $\mathrm{C}_{12}$ TEMPO rendered it difficult to access the solid surfaces, thereby reducing the film formation efficiency.
\end{abstract}

Keywords : Organic friction modifier, Head group, $\mathrm{C}_{12}$ TEMPO, Cyclic structure, Free radical, Boundary lubrication

\section{Introduction}

For achieving sustainable development in harmony with nature, it is crucial to establish a green lubrication that is environmentally friendly and can improve the energy efficiency by reducing the friction and wear in mechanical systems (Zhang, 2013). For example, it is estimated that about $23 \%$ of the total energy of the world is lost owing to tribological contacts, which can be sub-divided into $20 \%$ for friction and $3 \%$ for wear (Holmberg and Erdemir, 2017). One important approach of lubrication is to use low-viscosity base oils with a small amount of friction modifier 
additives (Spikes, 2015). The former can reduce the viscous resistance in the hydrodynamic and mixed lubrication regimes and the latter can prevent direct solid-solid contacts in the boundary and mixed lubrication regimes, thereby reducing the friction across the entire lubrication range (Zhang and Meng, 2015). Because the boundary lubrication regime, in which severe damage or even system failure is most likely to occur, extends to a wide range of operating conditions with constantly decreasing lubricant viscosity, the optimal design of friction modifier additives is of tremendous significance.

From the environmental perspective, organic friction modifiers (OFMs) that are sulfur and phosphorus-free and contain only carbon, hydrogen, oxygen, and nitrogen atoms are desirable to reduce hazardous emissions (Erdemir, 2005). The effectiveness of OFMs in reducing friction largely depends on their ability to become adsorbed from the lubricant solution on the solid surfaces and form a boundary film separating the opposing solid surfaces (Spikes, 2015; Desanker et al., 2017). Thus, the most typical molecular structure of an OFM consists of a hydrocarbon chain (tail group) and functional head group, such as carboxyl, amide, and glyceryl groups, at one end of the chain (Kenbeek et al., 2000). The functionality of the head groups is crucial because it determines the strength of the interactions with the solid surfaces and thereby, the adsorption ability of the OFMs. Literature shows that carboxylic acid and amine groups are generally more effective than alcohol, ester, nitrile, or halide groups on ferrous substrates (Spikes, 2015). Inheriting the feature of the molecular structure of fatty acids, which were the first OFM successfully used in 1918 (Spikes, 2015), the OFMs used thus far are basically slim molecules with small head groups. To explore the possibility of further performance improvement, a new type of OFM that features a large head group comprising of a nitrogen heterocycle with four nitrogen centers has been developed recently, and experimental results have demonstrated its excellent performance in reducing the friction and wear in the boundary lubrication regime (Desanker et al., 2017). This indicates that the effects of the head groups, including their size and shape, on the performance of OFMs are yet to be completely elucidated, and there is still a possibility to further optimize their design.

In this study, we synthesized N-(2,2,6,6-tetramethyl-1-oxyl-4-piperidinyl)dodecaneamide, which is characterized by a large and rigid cyclic head group containing a free radical, to explore its application as a new type of OFM. The synthesized compound will be simply referred to as $\mathrm{C}_{12}$ TEMPO hereafter. $\mathrm{C}_{12}$ TEMPO was selected as our target because we suppose that the unpaired electron in a free radical can interact strongly with metal surfaces and a rigid cyclic head group can bear heavy load. To clarify the effect of the head groups of OFMs, we also selected commercially available stearic acid and stearonitrile for a comparative experimental study. Their chemical structures are shown in Fig. 1. All the three types of OFMs have the same tail structure and approximately the same total length, but different head groups. In contrast to $\mathrm{C}_{12}$ TEMPO, stearic acid and stearonitrile possess small head groups without free radicals. As will also be confirmed from our experimental results presented in Section 3, stearic acid and stearonitrile are known to be excellent and poor OFMs, respectively (Spikes, 2015). Selection of these references allows for comprehensive understanding of various behaviors of OFMs. The frictional properties of the three types of OFMs added into a base oil were measured with a pin-on-disk type tribotester. Instead of fixing the sliding condition, we varied the sliding speed or normal load in the measurements. It will be discussed in Section 3 that this allowed us to understand the limits of the effective operating conditions of the OFMs and gain insight into the underlying mechanism of the effects of the head groups.

The paper is organized as follows. In Section 2, we describe the experimental details, including the materials and conditions of the friction tests. The results and discussion are presented in Section 3, and finally, the conclusions are summarized in Section 4.

(a)

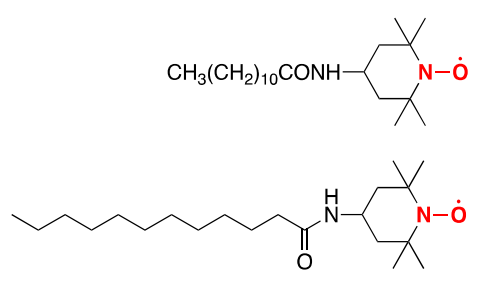

(b)

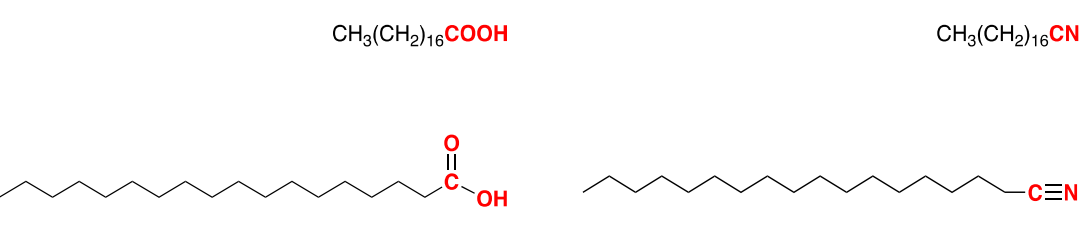

Fig. 1 Chemical structures of the OFMs used in this study: (a) $\mathrm{C}_{12}$ TEMPO, (b) stearic acid, and (c) stearonitrile. 


\section{Experimental details}

\subsection{Lubricants}

As described in Section 1, the synthesized $\mathrm{C}_{12}$ TEMPO and purchased stearic acid and stearonitrile were used as the OFMs. Their molecular weights are $353.57,284.48$, and $265.49 \mathrm{~g} / \mathrm{mol}$, respectively. The synthesis of $\mathrm{C}_{12} \mathrm{TEMPO}$ followed a literature procedure (Gallez et al., 1992). As shown in Fig. 2, the observed molecular ion peaks in the fast atom bombardment mass (FAB-MS) measurement were in good agreement with the calculated values \{calcd for $\mathrm{C}_{21} \mathrm{H}_{41} \mathrm{~N}_{2} \mathrm{O}_{2}[\mathrm{M}]^{+\cdot} 353$, found 353; calcd for $\mathrm{C}_{21} \mathrm{H}_{43} \mathrm{~N}_{2} \mathrm{O}_{2}[\mathrm{M}+2 \mathrm{H}]^{+} 355$, found 355 \}, confirming that the synthesized compound was our target molecule. It is noticed that the observed molecular ion peak changed with the elapsed time of the total ion current chromatogram. The nitroxyl radical was protonated gradually during ionization process. $[\mathrm{M}]^{+\bullet}$ was detected at $0.47 \mathrm{~min}$ (Fig. 2(a)), while $[\mathrm{M}+2 \mathrm{H}]^{+}$became the major peak at $1.99 \mathrm{~min}$ (Fig. 2(b)) and later. Elemental analysis found values for carbon, hydrogen, and nitrogen were within $0.4 \%$ of the calculated values for $\mathrm{C}_{12} \mathrm{TEMPO}$, indicating that the synthesized compound was pure enough for the following friction measurements. The elemental analysis data is as follows. Anal. Calcd for $\mathrm{C}_{21} \mathrm{H}_{41} \mathrm{~N}_{2} \mathrm{O}_{2}: \mathrm{C}, 71.34 ; \mathrm{H}, 11.69 ; \mathrm{N}, 7.92$. Found: $\mathrm{C}, 71.16 ; \mathrm{H}, 11.88 ; \mathrm{N}$, 7.86 .

(a)

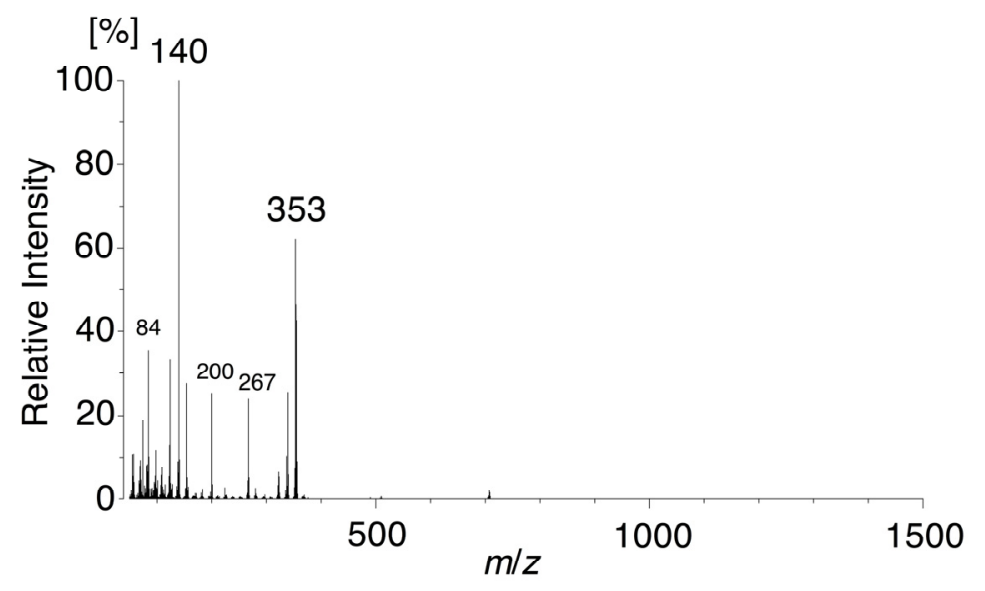

(b)

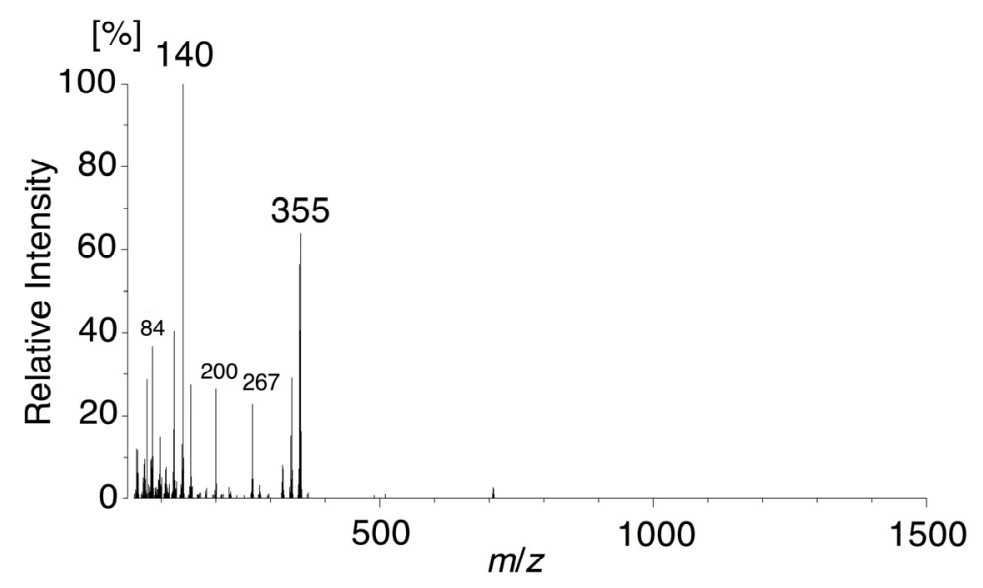

Fig. 2 FAB-MS spectra of $\mathrm{C}_{12}$ TEMPO measured at the elapsed time of (a) 0.47 and (b) 1.99 min of the total ion current chromatogram.

Polyalphaolefin oil with a viscosity of $6 \mathrm{cSt}$ at $100{ }^{\circ} \mathrm{C}$, simply referred to as PAO6 hereafter, was used as the base oil. For the friction measurements described in Section 2.2, the three types of OFMs were separately dissolved in the base oil, PAO6, at a concentration of $1 \mathrm{wt} . \%$ by ultrasonication and stirring for about $45 \mathrm{~min}$ and then oil-bath heating for about $15 \mathrm{~min}$ at a temperature of $65^{\circ} \mathrm{C}$. We visually confirmed that all the solutions were clear at the temperature of $40{ }^{\circ} \mathrm{C}$ used for friction measurements. It was reported that the standard concentration of friction modifiers in pratical applications can vary by up to 4 wt.\% (Kus and Kalin, 2019). The viscosity of PAO6 with and without 1 wt.\% OFMs is 
listed in Table 1; it was measured with a viscometer (TPE-100, Toki Sangyo) at the same temperature of $40{ }^{\circ} \mathrm{C}$ as in the friction measurements.

Table 1 Viscosity of the sample lubricants at $40{ }^{\circ} \mathrm{C}$.

\begin{tabular}{c|c}
\hline \hline Lubricant & Viscosity $[\mathrm{mPa} \cdot \mathrm{s}]$ \\
\hline Pure PAO6 & 23.9 \\
\hline PAO6 + 1 wt.\% $\mathrm{C}_{12}$ TEMPO & 25.1 \\
\hline PAO6 + 1 wt.\% stearic acid & 24.1 \\
\hline PAO6 + 1 wt.\% stearonitrile & 24.5 \\
\hline
\end{tabular}

\subsection{Friction measurements}

Friction measurements were conducted with a pin-on-disk type unidirectional tribotester, as shown in Fig. 3 . This equipment includes a rotating oil container and stationary pin. Spherical balls $8 \mathrm{~mm}$ in diameter were used as pins. Disks were fixed in the rotating container and immersed in the sample lubricants. Both the pins and disks were made of SUS304 stainless steel. The surface roughness was measured using an atomic force microscope (Dimension Icon, Bruker AXS) with a $20.0 \mu \mathrm{m} \times 20.0 \mu \mathrm{m}$ area for three pins and three disks. The averaged values of the arithmetic mean roughness $(\mathrm{Ra})$ and root-mean-square roughness $(\mathrm{Rq})$ were 15.1 and $21.4 \mathrm{~nm}$ for the pins and 0.76 and $1.33 \mathrm{~nm}$ for the disks, respectively.

In the measurements, the normal load applied to the pin was increased from 1.1 to $38.8 \mathrm{~N}$, corresponding to mean Hertzian contact pressure of 0.36 to $1.18 \mathrm{GPa}$ between the pin and disk. The sliding speed was increased by increasing the rotation speed of the oil container, $\omega$, from 10 to $100 \mathrm{rpm}$. As the radius of sliding tracks, $r$, was not always identical for different sample lubricants, the sliding speed ranged from 8.4 to $89.0 \mathrm{~mm} / \mathrm{s}$ in this study. For each sample lubricant, measurement was continuously conducted at the same sliding track when increasing either the normal load or sliding speed. The temperature was fixed at $40{ }^{\circ} \mathrm{C}$ with a heater embedded under the oil container. At each load and sliding speed condition, the friction forces were measured for 1 min with a sampling rate of $1.0 \mathrm{kHz}$ after a run-in period of $2 \mathrm{~min}$. For stable sliding, we confirmed that the fiction coefficient decreased with time and reached constant values during the run-in process of 2 min even at the minimum rotation speed of $10 \mathrm{rpm}$. However, this was not the case for unstable sliding. It is difficult to determine appropriate run-in time for unstable sliding. Considering that the major purpose is to clarify the effect of head groups of OFMs, we simply used 2 min for all the experiments, which still allows for comparison of different OFMs. Until specified otherwise, friction coefficients presented in Section 3 are the average value in the 1-min sampling period.

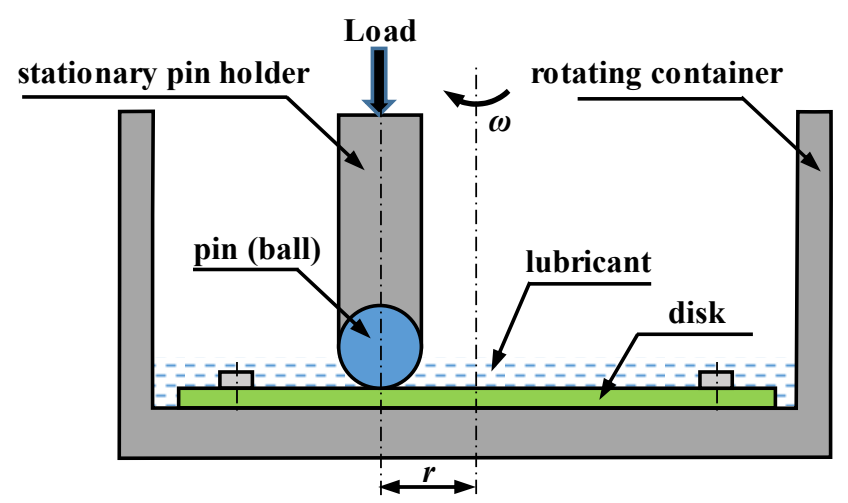

Fig. 3 Schematic of the pin-on-disk type tribotester.

To confirm the lubrication regime during our friction measurements, we calculated the lambda parameter defined below (Li et al., 2017; Liu et al., 2017).

$$
\Lambda=\frac{h_{\min }}{\sqrt{R_{\mathrm{qp}}^{2}+R_{\mathrm{qd}}^{2}}}
$$

where $h_{\min }$ is the theoretical minimum film thickness and $R_{\mathrm{qp}}$ and $R_{\mathrm{qd}}$ are the surface roughness of the pin and disk, 
respectively. The film thickness equations are available for four different regimes of the fluid film lubrication: isoviscous-rigid (IR), piezoviscous-rigid (PR), isoviscous-elastic (IE), and piezoviscous-elastic (PE) regimes (Yamamoto and Kaneta, 2010; Esfahanian and Hamrock, 1991). These regimes are distinguished by the dimensionless elasticity parameter, $g_{\mathrm{E}}$, and the dimensionless viscosity parameter, $g_{\mathrm{v}}$, defined below.

$$
\begin{aligned}
& g_{\mathrm{E}}=\frac{W^{8 / 3}}{U^{2}} \\
& g_{\mathrm{v}}=\frac{G W^{3}}{U^{2}} \\
& W=\frac{F}{E^{\prime} R^{2}} \\
& U=\frac{\eta_{0} u}{E^{\prime} R} \\
& G=\alpha E^{\prime} \\
& E^{\prime}=\frac{2}{\frac{1-v_{\mathrm{p}}^{2}}{E_{\mathrm{p}}}+\frac{1-v_{\mathrm{d}}^{2}}{E_{\mathrm{d}}}}
\end{aligned}
$$

where $W$ is a dimensionless load parameter, $U$ is a dimensionless speed parameter, $G$ is a dimensionless material parameter, $F$ is the applied normal load, $E^{\prime}$ is the composite elastic modulus of the pin and disk, $E_{\mathrm{p}}$ and $E_{\mathrm{d}}$ and $v_{\mathrm{p}}$ and $v_{\mathrm{d}}$ are the elastic moduli and Poisson's ratios of the pin and disk, respectively, $R$ is the radius in the sliding direction, $\eta_{0}$ is the lubricant viscosity at atmospheric pressure, $u$ is the mean velocity of the pin and disk in the sliding direction, and $\alpha$ is pressure-viscosity coefficient of the lubricant. With $E_{\mathrm{p}}=E_{\mathrm{d}}=200 \mathrm{GPa}, v_{\mathrm{p}}=v_{\mathrm{d}}=0.29, \alpha=13.5 \mathrm{GPa}^{-1}$ (Biresaw and Bantchev, 2013), viscosities of individual lubricants listed in Table 1 , and the normal loads and sliding speeds described above, $g_{\mathrm{E}}$ and $g_{\mathrm{v}}$ were calculated to be in the range of $4.4 \times 10^{6}-4.2 \times 10^{11}$ and $8.8 \times 10^{7}-2.8 \times 10^{13}$, respectively. These values correspond to the PE regime based on literature (Esfahanian and Hamrock, 1991) and hence, the following equation for the minimum film thickness in the PE regime was used (Desanker et al., 2017; Hamrock and Dowson, 1976a, 1976b, 1977; Li et al., 2017; Liu et al., 2017; Spikes, 2006; Yamamoto and Kaneta, 2010).

$$
h_{\min }=3.63 R U^{0.68} G^{0.49} W^{-0.073}\left(1-e^{-0.68 k}\right)
$$

where $k$ is a contact ellipticity parameter (equal to 1 in this study). Under all the normal load and sliding speed conditions in this study, the values of $h_{\min }$ and $\Lambda$ were less than $8.58 \mathrm{~nm}$ and 0.40 , respectively. Here values of $\Lambda$ were calculated using surface roughness measured before friction tests rather than the increased surface roughness after friction tests. The small values of calculated $\Lambda$ confirm that all the contacts during our friction measurements were in the boundary lubrication regime.

\section{Results and discussion}

Figure 4 shows the change in the dynamic friction coefficient with increasing sliding speed for pure PAO6 and the solutions of the three types of OFMs. The normal load was fixed at a relatively low value of $1.5 \mathrm{~N}$ (i.e., mean Hertzian pressure of $0.40 \mathrm{GPa}$ ) during the measurements. All the OFM solutions exhibited much smaller friction coefficients than the pure base oil, particularly at low sliding speeds. This demonstrates the effectiveness of these OFMs in improving the performance of the boundary lubrication as compared to pure PAO6. A decrease in friction coefficient with sliding speed is observed for pure PAO6. This might be attributed to running-in rather than effect of fluid lubrication, because we confirmed from $\Lambda$ values that all the friction measurements were in the boundary lubrication regime as described in Section 2. Comparing the results of the three types of OFMs, we can notice difference between them. As the sliding speed increases, the friction coefficient remains nearly constant at approximately 0.12 for stearic acid, whereas it decreases initially and then converges to approximately 0.13 for $\mathrm{C}_{12}$ TEMPO. Although the friction coefficient of stearonitrile also shows a decreasing trend with increasing sliding speed, it fluctuates apparently in contrast to the stable behaviors of stearic acid and $\mathrm{C}_{12}$ TEMPO. In the boundary lubrication regime, the friction 
coefficients are determined by the surface coverage of the boundary films preventing direct solid-solid contacts. Therefore, the different behaviors observed in Fig. 4 demonstrate that stearic acid can rapidly form an effective boundary film even at low sliding speeds, whereas it is difficult for $\mathrm{C}_{12}$ TEMPO and stearonitrile. Extensive studies demonstrate that as an OFM stearic acid is highly effective compared to stearonitrile (Beltzer and Jahanmir, 1988; Jahanmir and Beltzer, 1986; Spikes, 2015), and hence, the following discussion will focus on $\mathrm{C}_{12}$ TEMPO while comparing with stearic acid and stearonitrile.

To form boundary films, the head groups of OFMs must first approach and then interact with the solid surfaces. Therefore, the ability and efficiency of the boundary film formation is associated with not only the interaction strength between the head groups of the OFMs and solid surfaces, but also with the accessibility of the head groups to the solid surfaces. Because the head group of $\mathrm{C}_{12}$ TEMPO is large and rigid, its accessibility to solid surfaces should be less than that of stearic acid and stearonitrile, which have small head groups. From Fig. 4, although it is difficult to compare the interaction strengths of $\mathrm{C}_{12}$ TEMPO and stearic acid, we can infer that the interaction strength with steel surfaces is much stronger for $\mathrm{C}_{12}$ TEMPO than that for stearonitrile. We suggest that, once approaching steel surfaces, the head group of $\mathrm{C}_{12}$ TEMPO will become adsorbed on the surfaces and be barely removed by sliding. Consequently, as time elapses during the measurements with increasing sliding speed, more $\mathrm{C}_{12}$ TEMPO molecules will approach and become adsorbed on the steel surfaces, resulting in a steady decrease in friction coefficient in contrast to the fluctuation behavior in the case of stearonitrile, as seen in Fig. 4. Specifically, $\mathrm{C}_{12}$ TEMPO has a better ability to form effective boundary films than stearonitrile owing to its strong surface interaction, but its film formation efficiency is lower than that of stearic acid because of its less surface accessibility. The efficiency to form stable and effective boundary films on the steel disks follows the order: stearic acid $>\mathrm{C}_{12}$ TEMPO $>$ stearonitrile.

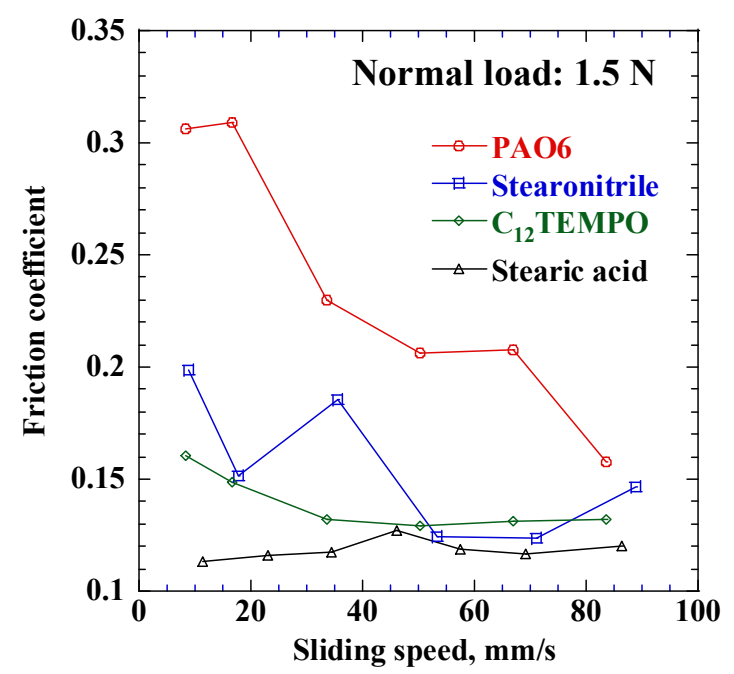

Fig. 4 Change in the dynamic friction coefficient with increasing sliding speed for pure PAO6 and 1 wt.\% solutions of $\mathrm{C}_{12}$ TEMPO, stearic acid, and stearonitrile in PAO6. A low normal load of $1.5 \mathrm{~N}$ was applied and the corresponding Hertzian contact pressure was $0.40 \mathrm{GPa}$.

Figure 5 shows the change in the friction force with increasing normal load for the solutions of the three types of OFMs in PAO6. At the sliding speed of $68.0 \mathrm{~mm} / \mathrm{s}$, as shown in Fig. 5(a), the friction coefficient of $\mathrm{C}_{12}$ TEMPO remains stable at small values in a wide range of normal load and beyond a critical normal load of $29.2 \mathrm{~N}$ (i.e., mean Hertzian pressure of $1.08 \mathrm{GPa}$ ) the friction coefficient exhibits a sharp increase. In contrast, the friction coefficient of stearic acid shows fluctuation from the normal load of $6.0 \mathrm{~N}$ (i.e., mean Hertzian pressure of $0.64 \mathrm{GPa}$ ) and an obvious increase from $11.8 \mathrm{~N}$ (i.e., mean Hertzian pressure of $0.80 \mathrm{GPa}$ ). Same as in Fig. 4, the friction coefficient of stearonitile is apparently unstable, showing large fluctuation at low normal loads and a sharp increase from $9.9 \mathrm{~N}$ (i.e., mean Hertzian pressure of $0.75 \mathrm{GPa}$ ). Therefore, the critical normal load for $\mathrm{C}_{12} \mathrm{TEMPO}$ is evidently the largest, nearly thrice that for stearic acid and stearonitrile. This demonstrates that, not stearic acid, but $\mathrm{C}_{12}$ TEMPO has the highest load-carrying capability. Specifically, $\mathrm{C}_{12}$ TEMPO can form the strongest boundary film, which is difficult to be removed by the high friction in a heavy-loaded sliding contact. This may be ascribed to two reasons. One is that the free radical of $\mathrm{C}_{12}$ TEMPO may interact with steel surfaces more strongly than the carboxylic acid group of stearic acid, 
and the other is that the large and rigid head group of $\mathrm{C}_{12}$ TEMPO may help in enduring the normal load. The former needs to be confirmed by further experiments or quantum chemical calculations. However, as the carboxylic acid group is known to show considerably strong interaction (Beltzer and Jahanmir, 1988; Jahanmir and Beltzer, 1986; Loehlé et al., 2015; Spikes, 2015), we suggest that the large size and rigidity of the head group of $\mathrm{C}_{12}$ TEMPO may play an important role in enhancing its load-carrying capacity.

At the sliding speed of $26.7 \mathrm{~mm} / \mathrm{s}$, a tendency in Fig. 5(b) similar to that in Fig. 5(a) can be observed. The critical normal load of $\mathrm{C}_{12}$ TEMPO is $21.5 \mathrm{~N}$, larger than those of stearic acid and stearonitrile. This again demonstrates that $\mathrm{C}_{12}$ TEMPO possesses the highest load-carrying capability. However, the critical normal loads for $\mathrm{C}_{12} \mathrm{TEMPO}$ and stearonitrile at the sliding speed of $26.7 \mathrm{~mm} / \mathrm{s}$ are evidently smaller than those at $68.0 \mathrm{~mm} / \mathrm{s}$. In contrast, the critical normal load for stearic acid remains nearly unchanged with the sliding speed. As the sliding speed decreases, the amount of OFMs entrained into the contacting interface decreases. Thus, we suggest that the low efficiency of $\mathrm{C}_{12}$ TEMPO and poor ability of stearonitrile to form effective boundary films, as discussed above, should be the reasons for the decrease in the critical normal loads of $\mathrm{C}_{12}$ TEMPO and stearonitrile at a low sliding speed.

(a)

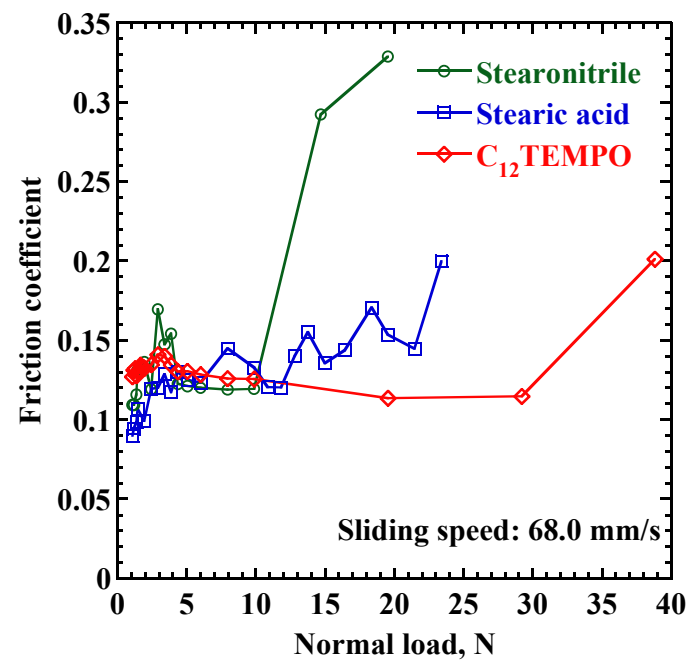

(b)

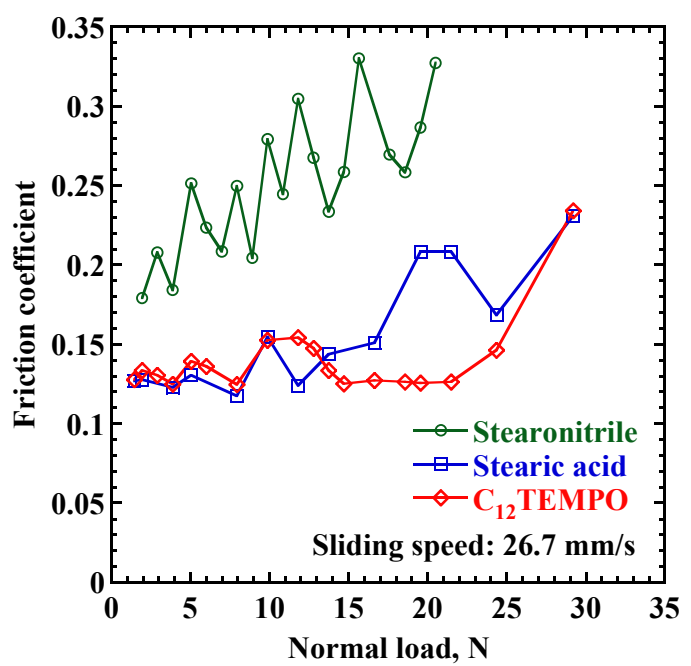

Fig. 5 Change in the friction coefficient with increasing normal load for $1 \mathrm{wt} . \%$ solutions of $\mathrm{C}_{12}$ TEMPO, stearic acid, and stearonitrile in PAO6. The sliding speed was fixed as (a) 68.0 and (b) $26.7 \mathrm{~mm} / \mathrm{s}$.

Comparing $\mathrm{C}_{12} \mathrm{TEMPO}$ with stearic acid at normal loads smaller than the critical values, we found that the friction coefficient of $\mathrm{C}_{12}$ TEMPO is slightly larger than stearic acid at the sliding speed of $68.0 \mathrm{~mm} / \mathrm{s}$ (Fig. $5(\mathrm{a})$ ) but there is little difference at the sliding speed of $26.7 \mathrm{~mm} / \mathrm{s}$ (Fig. 5(b)). At the sliding speed of $68.0 \mathrm{~mm} / \mathrm{s}$, the instantaneous friction coefficients of $\mathrm{C}_{12}$ TEMPO and stearic acid measured in the 1-min sampling period at the normal loads of 2.9 and $5.0 \mathrm{~N}$ (i.e., mean Hertzian pressures of 0.50 and $0.60 \mathrm{GPa}$ ) are compared in Figs. 6(a) and 6(b), respectively. In contrast to the large fluctuation observed for stearic acid, $\mathrm{C}_{12}$ TEMPO exhibits stable instantaneous friction coefficient. Such stable frictional behavior of $\mathrm{C}_{12}$ TEMPO is observed at normal loads up to the critical value of $29.2 \mathrm{~N}$, as presented in Fig. 6(c). This comparison suggests again that the head group of $\mathrm{C}_{12}$ TEMPO helps to form boundary film more robust than stearic acid.

\section{Conclusion}

We synthesized $\mathrm{C}_{12}$ TEMPO and compared its pin-on-disk friction properties with those of stearic acid and stearonitrile, to explore its application as a new type of OFM and also clarify the effect of the head groups of OFMs. By varying the normal load or sliding speed, we found that the OFMs, which had similar overall length and a long alkyl chain but different head groups, exhibited different effective ranges of the operating conditions. $\mathrm{C}_{12}$ TEMPO showed the highest load-carrying capacity, up to nearly thrice that of stearic acid and stearonitrile. Moreover, even at small normal loads, the instantaneous friction coefficient of $\mathrm{C}_{12}$ TEMPO was obviously more stable than that of stearic acid. These results suggested that $\mathrm{C}_{12}$ TEMPO can form an effective boundary film that is robust to heavy-loaded sliding. From the results obtained when varying the sliding speed, we inferred that the efficiency to form the boundary film followed the 
order: stearic acid $>\mathrm{C}_{12}$ TEMPO $>$ stearonitrile. The strong surface interaction owing to the free radical and rigidity owing to the cyclic structure of the head group were expected to be the reasons for the stable frictional behavior and high load-carrying capacity of $\mathrm{C}_{12}$ TEMPO. However, the large size of the head group possibly reduced its accessibility to the solid surfaces, thereby reducing the efficiency of the boundary film formation. This study was the first trial to incorporate a rigid cyclic structure with a free radical as the head group of OFMs, and the findings of this study are expected to motivate future research on the optimal molecular design of OFMs.

(a)

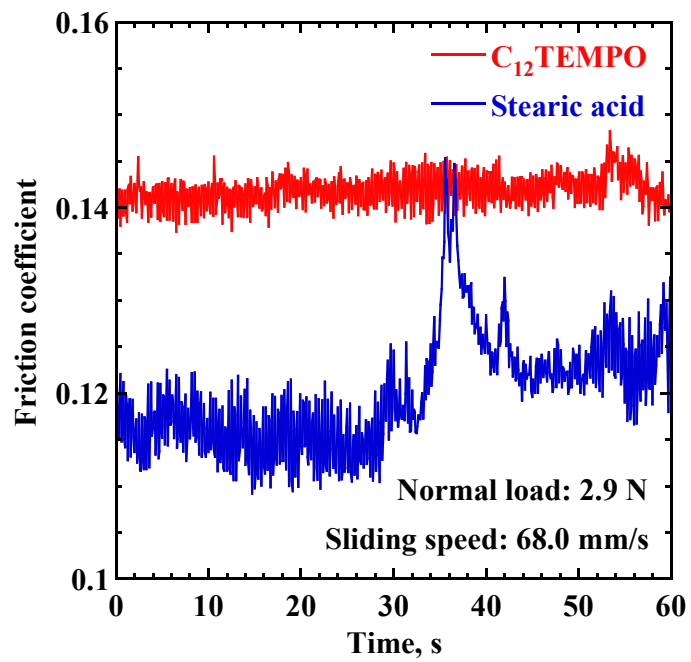

(b)

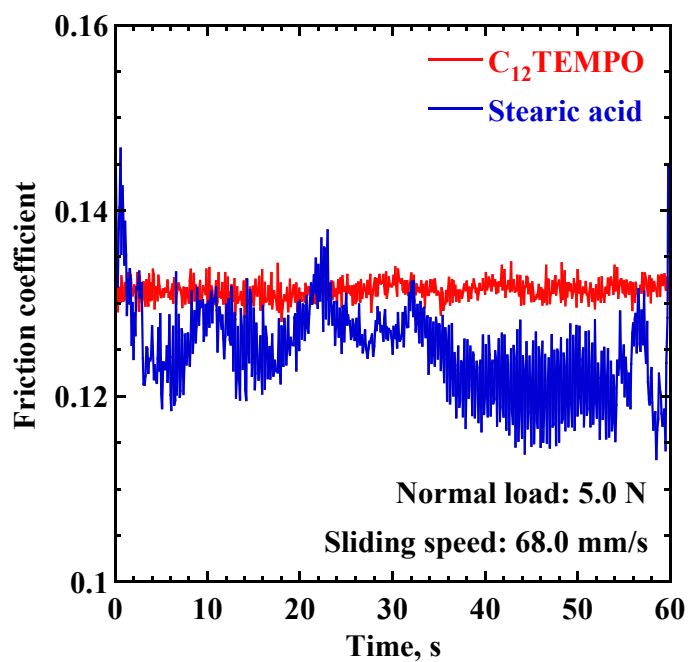

(c)

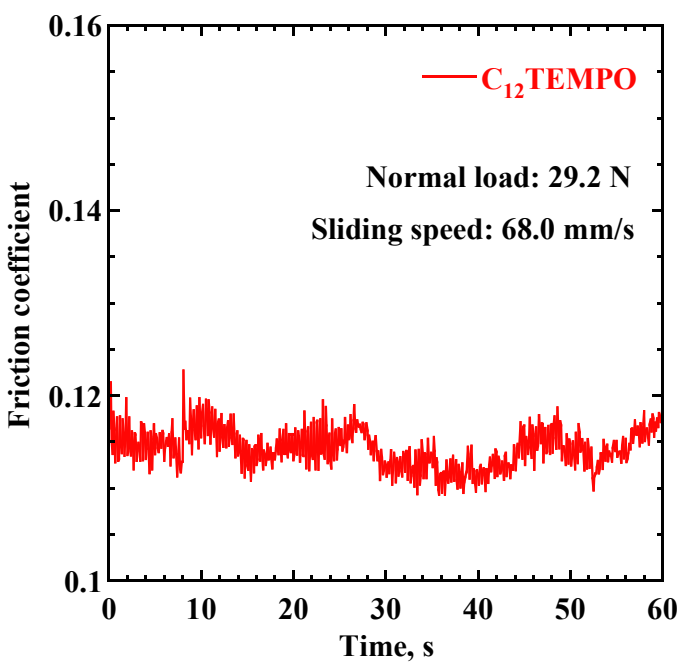

Fig. 6 Comparison of instantaneous friction coefficients measured in 1 min for $1 \mathrm{wt} \%$ solutions of $\mathrm{C}_{12} \mathrm{TEMPO}$ and stearic acid in PAO6 at sliding speed of $68.0 \mathrm{~mm} / \mathrm{s}$ and normal loads of (a) 2.9, (b) 5.0, and (c) $29.2 \mathrm{~N}$. Data for stearic acid in (c) is absent because the normal load is beyond the critical load of stearic acid.

\section{Acknowledgments}

This work was supported in part by the JSPS Grant-in-Aid for Scientific Research (B No. 17H03164). X. Zhang is grateful to Guangdong University of Technology for the financial support through the program of Study Abroad for Scholar in Guangdong University of Technology. We also thank Dr. Kin-ichi Oyama (Research Center for Materials Science, Nagoya University) for conducting the mass spectrometry measurements and the elemental analysis.

\section{References}

Beltzer, M. and Jahanmir, S., Effect of additive molecular structure on friction, Lubrication Science, Vol. 1, No. 1 (1988), pp. 3-26, DOI: 10.1002/ls.3010010103. 
Xiaowei Zhang, Tsukamoto, Hedong Zhang, Mitsuya, Itoh and Fukuzawa, Journal of Advanced Mechanical Design, Systems, and Manufacturing, Vol.14, No.4 (2020)

Biresaw, G. and Bantchev, G. B., Pressure viscosity coefficient of vegetable oils, Tribology Letters, Vol. 49, No. 3 (2013), pp. 501-512, DOI: 10.1007/s11249-012-0091-9.

Desanker, M., He, X., Lu, J., Liu, P., Pickens, D. B., Delferro, M., Marks, T. J., Chung, Y. W. and Wang, Q. J., Alkyl-cyclens as effective sulfur- and phosphorus-free friction modifiers for boundary lubrication, ACS Applied Materials \& Interfaces, Vol. 9, (2017), pp. 9118-9125, DOI: 10.1021/acsami.6b15608.

Esfahanian, M. and Hamrock, B. J., Fluid-film lubrication regimes revisited, Tribology Transactions, Vol. 34, No. 4 (1991), pp. 628-632, DOI: 10.1080/10402009108982081.

Erdemir, A., Review of engineered tribological interfaces for improved boundary lubrication, Tribology International, Vol. 38, (2005), pp. 249-256, DOI: 10.1016/j.triboint.2004.08.008.

Gallez, B., Demeure, R., Debuyst, R., Leonard, D., Dejehet, F. and Dumont, P., Evaluation of nonionic nitroxyl lipids as potential organ-specific contrast agents for magnetic resonance imaging, Magnetic Resonance Imaging, Vol. 10, No. 3 (1992), pp. 445-455, DOI: 10.1016/0730-725X(92)90516-3.

Hamrock, B. J. and Dowson, D., Isothermal elastohydrodynamic lubrication of point contacts: Part I-Theoretical formulation, Journal of Lubrication Technology, Vol. 98, (1976a), pp. 223-229, DOI: 10.1115/1.3452801.

Hamrock, B. J. and Dowson, D., Isothermal elastohydrodynamic lubrication of point contacts: Part II-Ellipticity parameter results, Journal of Lubrication Technology, Vol. 98, (1976b), pp. 375-383, DOI: 10.1115/1.3452861.

Hamrock, B. J. and Dowson, D., Isothermal elastohydrodynamic lubrication of point contacts: Part III. Fully flooded results, Journal of Lubrication Technology, Vol. 99, (1977), pp. 264-276, DOI: 10.1115/1.3453074.

Holmberg, K. and Erdemir, A., Influence of tribology on global energy consumption, costs and emissions, Friction, Vol. 5, No. 3 (2017), pp. 263-284, DOI: 10.1007/s40544-017-0183-5.

Jahanmir, S. and Beltzer, M., Effect of additive molecular-structure on friction coefficient and adsorption, Journal of Tribology-Transactions of the ASME, Vol. 108, (1986), pp. 109-116, DOI:10.1115/1.3261129.

Kenbeek, D., Buenemann, T. and Rieffe, H., Review of organic friction modifiers -contribution to fuel efficiency? International Spring Fuels \& Lubricants Meeting \& Exposition, (2000), DOI: 10.4271/2000-01-1792.

Kus, M. and Kalin, M., Influence of additives and their molecular structure on the static and dynamic wetting of oil on steel at room temperature, Applied Surface Science, Vol. 490, (2019), pp. 420-429, DOI: 10.1016/j.apsusc.2019.06.111.

Li, X., Deng, X., Kousaka, H. and Umehara, N., Comparative study on effects of load and sliding distance on amorphous hydrogenated carbon (a-C:H) coating and tetrahedral amorphous carbon (ta-C) coating under base-oil lubrication condition, Wear, Vol. 392-393, (2017) pp. 84-92, DOI: 10.1016/j.wear.2017.09.009.

Liu, X., Yamaguchi, R., Umehara, N., Deng, X., Kousaka, H. and Murashima, M., Clarification of high wear resistance mechanism of ta-CNx coating under poly alpha-olefin (PAO) lubrication, Tribology International, Vol. 105, (2017), pp. 193-200, DOI: 10.1016/j.triboint.2016.10.016.

Loehlé, S., Matta, C., Minfray, C., Mogne T. Le, Iovine, R., Obara, Y., Miyamoto, A. and Martin, J. M., Mixed lubrication of steel by C18 fatty acids revisited. PartI: toward the formation of carboxylate, Tribology International, Vol. 82, (2015), pp. 218-227, DOI: 10.1016/j.triboint.2014.10.020.

Spikes, H. A., Sixty years of EHL, Lubrication Science, Vol. 18, (2006), pp. 265-291, DOI: 10.1002/1s.23.

Spikes, H., Friction modifier additives, Tribology Letters, Vol. 60, No. 1 (2015), 5, DOI: 10.1007/s11249-015-0589-z.

Yamamoto, Y. and Kaneta, Y., Tribology, 2nd ed., Ohmsha Press: Tokyo, 2010 (in Japanese).

Zhang, J. and Meng, Y., Boundary lubrication by adsorption film, Friction, Vol. 3, No. 2 (2015), pp. 115-147, DOI 10.1007/s40544-015-0084-4.

Zhang, S. W., Green tribology: fundamentals and future development, Friction, Vol. 1, No. 2 (2013), pp. 186-194, DOI: 10.1007/s40544-013-0012-4. 